See discussions, stats, and author profiles for this publication at: https://www.researchgate.net/publication/220956262

\title{
Linking search tasks with low-level eye movement patterns
}

Conference Paper · January 2010

DOI: 10.1145/1962300.1962323 · Source: DBLP

\section{CITATIONS}

18

7 authors, including:

$$
\text { Michael J. Cole }
$$

Rutgers, The State University of New Jersey

45 PUBLICATIONS 957 CITATIONS

SEE PROFILE

Ralf Bierig

18. National College of Ireland

44 PUBLICATIONS 414 CITATIONS

SEE PROFILE
READS

140

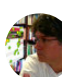

Jacek Gwizdka

University of Texas at Austin

165 PUBLICATIONS 2,740 CITATIONS

SEE PROFILE

(25) Nicholas J. Belkin

Rutgers, The State University of New Jersey

221 PUBLICATIONS 9,518 CITATIONS

SEE PROFILE

Some of the authors of this publication are also working on these related projects:

Multidimensional relevance modeling via psychometrics and crowdsourcing View project

Neurophysiological correlates of information relevance View project 


\title{
Linking Search Tasks with Low-level Eye Movement Patterns
}

\author{
Michael J. Cole, Jacek Gwizdka, Ralf Bierig, Nicholas J. Belkin, Jingjing Liu, Chang Liu, \\ Xiangmin Zhang \\ School of Communication and Information \\ Rutgers, The State University of New Jersey \\ 4 Huntington Street, New Brunswick, NJ 08901, USA \\ \{m.cole,jacekg,belkin,bierig\}@rutgers.edu, \{jingjing, changl\}@eden.rutgers.edu, xiangminz@gmail.com
}

\begin{abstract}
Motivation - On-the-task detection of the task type and task attributes can benefit personalization and adaptation of information systems.
\end{abstract}

Research approach - A web-based information search experiment was conducted with 32 participants using a multi-stream logging system. The realistic tasks were related directly to the backgrounds of the participants and were of distinct task types.

Findings/Design - We report on a relationship between task and individual reading behaviour. Specifically we show that transitions between scanning and reading behaviour in eye movement patterns are an implicit indicator of the current task.

Research limitations/Implications - This work suggests it is plausible to infer the type of information task from eye movement patterns. One limitation is a lack of knowledge about the general reading model differences across different types of tasks in the population. Although this is an experimental study we argue it can be generalized to real world text-oriented information search tasks.

Originality/Value - This research presents a new methodology to model user information search task behaviour. It suggests promise for detection of information task type based on patterns of eye movements.

Take away message - With increasingly complex computer interaction, knowledge about the type of information task can be valuable for system personalization. Modelling the reading/scanning patterns of eye movements can allow inference about the task type and task attributes.

\section{Keywords}

Personalization; cognitive task; interactive information retrieval; information search; user models; eye movements; user study

\section{INTRODUCTION}

Extended interaction with an information system to obtain and utilize information is a prime example of a complex cognitive task. Understanding the cognitive aspects of information seeking to improve user interfaces and better 'fit' information systems to individual users to improve their efficiency and effectiveness is an important direction for research (Belkin, 2008).

A specific goal is to build personalized systems that exploit the task characteristics to improve the effectiveness of search and information retrieval (IR) (Kelly \& Belkin, 2004; Liu \& Belkin, 2010; White \& Kelly, 2006). One way to build better adaptive systems is to construct richer user models based on inferences about the cognitive state of the user. Eye tracking information can inform user models and enhance performance by including cognitive features related to user attention and intent (Conati et al., 2005). Connecting user situation aspects, e.g. task characteristics, with unobtrusive measurements of user cognitive strategies is a specific way to adapt information systems to the user's goal.

Acquisition of eye data for use by IR systems has been investigated to detect relevance (Buscher et al., 2008a, Oliveira, et al. 2009) and provide real-time relevance feedback for query expansion (Buscher et al., 2008b). Our work links user eye movement patterns to the type of search task performed. In this paper we present evidence showing a correlation between patterns of lexical eye movement and user search tasks.

\section{BACKGROUND AND RELATED WORK}

\section{Task Effects on Search Behaviour}

One aspect of user context that affects information search behaviour is the nature of the user's task. The effects of task characteristics, including complexity, difficulty, and stage, on search behaviour, including usefulness and relevance judgments, have been studied extensively (e.g. Byström \& Järvelin, 1995; White \& Kelly, 2006; Kelly \& Belkin, 2004; Li, 2009).

Eye tracking metrics such as fixation duration, number of fixations, pupil diameter, etc., have been used as evidence of user engagement and to study patterns of eye movements associated with reading behaviours. Farzan and Brusilovsky (2009) used eye tracking to explore the usefulness of social navigation clues to users performing web searches. Document level patterns have been identified, e.g. an "F" shape reading pattern for a search engine result page (SERP) (Sherman, 2005; Lorigo et al., 2008). Lorigo et al. (2008) examined the number of fixations, fixation duration, and time spent 
on tasks for Google and Yahoo! Eye and eye movement data across different IR activities has been investigated by looking at SERP interaction during informational and navigational tasks (Granka et al., 2004; Lorigo et al., 2008; Guan and Cutrell, 2007; Terai et al., 2008).

\section{Eye Movements and Visual Cognition Strategies}

Eye fixations provide information about attentional states because the only way to acquire information visually is by repeated eye gaze on a location. Coupling behaviour, such as effects of user's decisions, with inferences from these fixation observations, for example with words that are lexically processed, is one way to discover user intent during task performance.

Eye movements are cognitively controlled and visual information processing is affected by immediate task properties in reading, face processing, scene processing and visual search (Rayner et al., 1998; Rayner et al., 2009; Torralba et al., 2006; Findlay \& Gilchrist, 2003). It is hypothesized that different visual cognition strategies are employed to meet the requirements for each type of task such as the encoding of appropriate information features for the task (Rayner et al., 2009). There is some understanding of the probable mechanism by which these types of task differences cause changes in fixation patterns (Hayhoe et al., 2007). With the notable exception of reading, eye movement behaviour for fixations and saccade distances has been found to be similar for low-level visual tasks across individuals and cultural groups (Rayner et al., 2007).

This suggests that extended information processing interactions in service of a task may involve selection of problem solving strategies and tactics that condition parameters of the visual cognition system used to control eye movements. In this way the user task could affect low-level information gathering processes. Visual information acquisition is important for information seeking, for example in reading to process texts. Taking account of task effects on visual cognition, for example through effects on reading eye movement control, may therefore be a valuable component in user models to personalize search and information retrieval.

\section{Objectives and Research Questions}

Our goal is to predict the task type based on the detected searcher behaviour during a search session. This work examines eye movement patterns with respect to a detailed classification of search tasks (Li, 2009). One goal is to learn if eye movement measures can be used as implicit evidence that users are engaged in particular types of tasks, and so enable adaptive personalization. To address this idea, we conducted a user study with realistic and well-defined Web search tasks, without constraint on the information sources or search engines.

We explored two specific questions. 1) Do the typical parameters of the reading behaviour model vary across individuals when they perform the same tasks? 2) Do the parameters of the models of participant's reading behaviour change due to the different task types?

\section{METHODOLOGY}

\section{Experimental Procedure}

The user study investigated behaviours associated with different task types for 32 undergraduate journalism students carrying out realistic professional journalism tasks. Each participant was given a tutorial and performed four tasks involving web search (described below). Participants were asked to continue searching until they had gathered enough information to accomplish the task or 20 minutes had elapsed. During the search, all of the participants' interactions with the computer system, including eye gaze, were logged.

\section{Tasks}

Our study concerned the work domain of journalism because it can be associated with any topic, yet has a small number of task types. A set of four tasks was identified by interviewing journalism faculty and practicing journalists. The tasks were designed to vary according to values of the characteristics which we believed could affect search behaviour (Li, 2009).

After a training task, participants completed four tasks in counterbalanced order: advanced obituary (AO), interview preparation (IP), copyediting (CE), and background information (BI). The tasks varied in several dimensions: complexity defined as the number of necessary steps needed to achieve the task goal (for example, identifying an expert and then finding their contact information), the task product (factual vs. intellectual, e.g., fact checking vs. production of a document), the information object (a complete document vs. a document segment), and the nature of the task goal (specific vs. amorphous). In Table 1, one can see the advanced obituary and the copy editing tasks have the least similarity.

Table 1: Task characteristics

\begin{tabular}{|c|c|c|c|c|}
\hline Task & Product & Level & Goal & Complexity \\
\hline AO & F, I & Document & Specific & High \\
\hline CE & F & Segment & Specific & Low \\
\hline IP & F, I & Document & Mixed & Low \\
\hline BI & F, I & Document & Amorphous & High \\
\hline \multicolumn{5}{|c|}{ F = Factual I=Intellectual }
\end{tabular}

\section{Reading Models}

In most previous eye tracking work in information search settings reports of reading behaviour have been based on analysis of eye gaze position aggregates ('hot spots'), without distinguishing the fixation subsequences that comprise true reading behaviour. The eye fixation analysis in our work is based on our implementation of the E-Z Reader reading model (Reichle et al., 2006). The inputs are the fixation location and its duration ( $>113 \mathrm{~ms}$, which is the threshold for lexical processing (Reingold \& Rayner, 2006). The algorithm was used to distinguish reading fixation sequences from isolated fixations, which we define as 'scanning' fixations. 
Scanning fixations provide some semantic information, limited to that available in the foveal (in focus) visual field (Rayner \& Fischer, 1996). Fixations in a reading sequence provide more information because information is gained from the larger parafoveal region (Rayner et al., 2003), and because of the richer semantic structure available in compositions of text, including sentences, paragraphs, etc., as compared to isolated units of several words. Importantly, some of the types of semantic information available through reading sequences may be crucial for satisfying of user task requirements.

The reading model algorithm was used to classify fixations as reading or scanning. The relationship of fixations within a reading sequence further identifies a fixation as Start Reading, Read (i.e. continue reading), or End Reading. This classification of fixations was used to create reading state transition models (see an example in Figure 1) for each pair (participant and task).

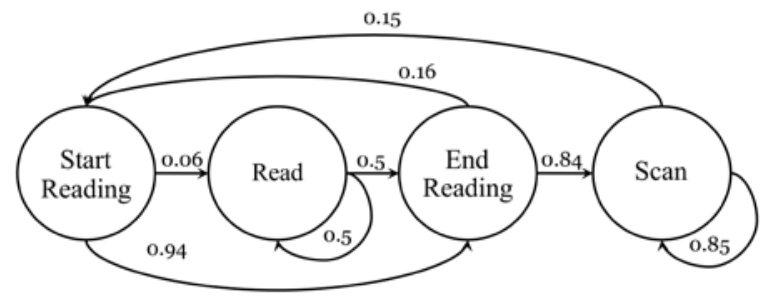

Figure 1: One participant's reading state transition model (AO task session).

A user's decision to read or scan is a distinctive cognitive commitment. Our analysis focuses on the Scan to Start Reading, and the End Reading to Scan transition probabilities. These decisions reflect the visual information acquisition strategy adopted by the user to achieve their task goal.

\section{Data Collection}

We used a multi-source logging system (Bierig et al., 2009). Eye data was collected with a Tobii T-60 eyetracker (1280x1024@60 Hz). We used eye fixation data as calculated by the Tobii Studio software (foveal radius $=35$ pixels).

Logging issues for two participants prevented data analysis of all four tasks for those participants. In the following we report on results for 30 of the 32 participants in the study.

\section{RESULTS}

\section{Individual Biases in Reading Models}

An individual may have a general bias towards reading behaviour and so their typical reading model parameters would differ from someone with a general bias towards scanning. Figure 2 shows the differences between individuals for reading model state transition probabilities. For each participant, it shows the range of the reading model state transition probabilities that reflect a decision to switch from scanning to reading (Scan to Start Reading) or to switch back to scanning rather than continue reading (End Reading to Scan). Figure 2 also shows the absolute differences in individual biases to make these decisions to switch. Note that the participants have been ordered by the mean transition probability measured over all of their tasks. One can see there are variations in the absolute values of the Scan to Start Reading parameter. When all participants are considered, it appears there is both a similar range of differences in absolute values and the range of individual variability for the Scan to Start Reading and End Reading to Scan transition probabilities. Further, one can see from Figure 2 that for many participants, a higher absolute mean for the End Reading to Scan transition probability is matched to a lower absolute mean for the Scan to Start Reading transition probability as compared to the rest of the participants. For example, participant s010 has the second highest mean for the End Reading to Scan state transition probability, but is the second lowest for the Scan to Start Reading transition probability. Participant s003 has the lowest absolute mean for the End Reading to Scan transition probability but has the highest mean for the Scan to Start Reading transition probability. There is no analytic reason to expect this pattern in the data. As Figure 1 shows, a user makes a choice at the end of a reading sequence of eye fixations, for example at the end of a line of text. They can continue reading by starting a new sequence of reading fixations, for example by reading the next line of text or jumping to a different place in a document but immediately begin reading. The alternative is to decide to scan (both reading and scanning, as defined here, are lexical and in each case the user can acquire information from the text they fixated on). In short, there is no reason to suppose a person's reading model biases could not include both a tendency to switch from scanning to reading and a bias to switch to scanning from reading. Figure 2 provides evidence that the participants were relatively consistent in their bias towards reading or towards scanning. Those who were inclined to switch to reading from scanning were also inclined to continue reading as compared to others. In contrast, there were other participants generally biased towards scanning in the same way.

In summary, Figure 2 provides evidence for clear individual differences in reading models in several ways. First, there are absolute differences in mean transition values. Second, differences exist in the individual variability in reading behaviour. We observed a similar distribution of mean values for the Scan to Start Reading and End Reading to Scan transition probabilities for individuals. Finally, there seems to be evidence for consistent individual bias towards reading or scanning. Taken together, these observations confirm a positive answer to our first research question regarding the existence of individual differences in reading models.

Task Influence on an Individual's Reading Model Our second research question asked whether participants respond consistently to the task treatments. 


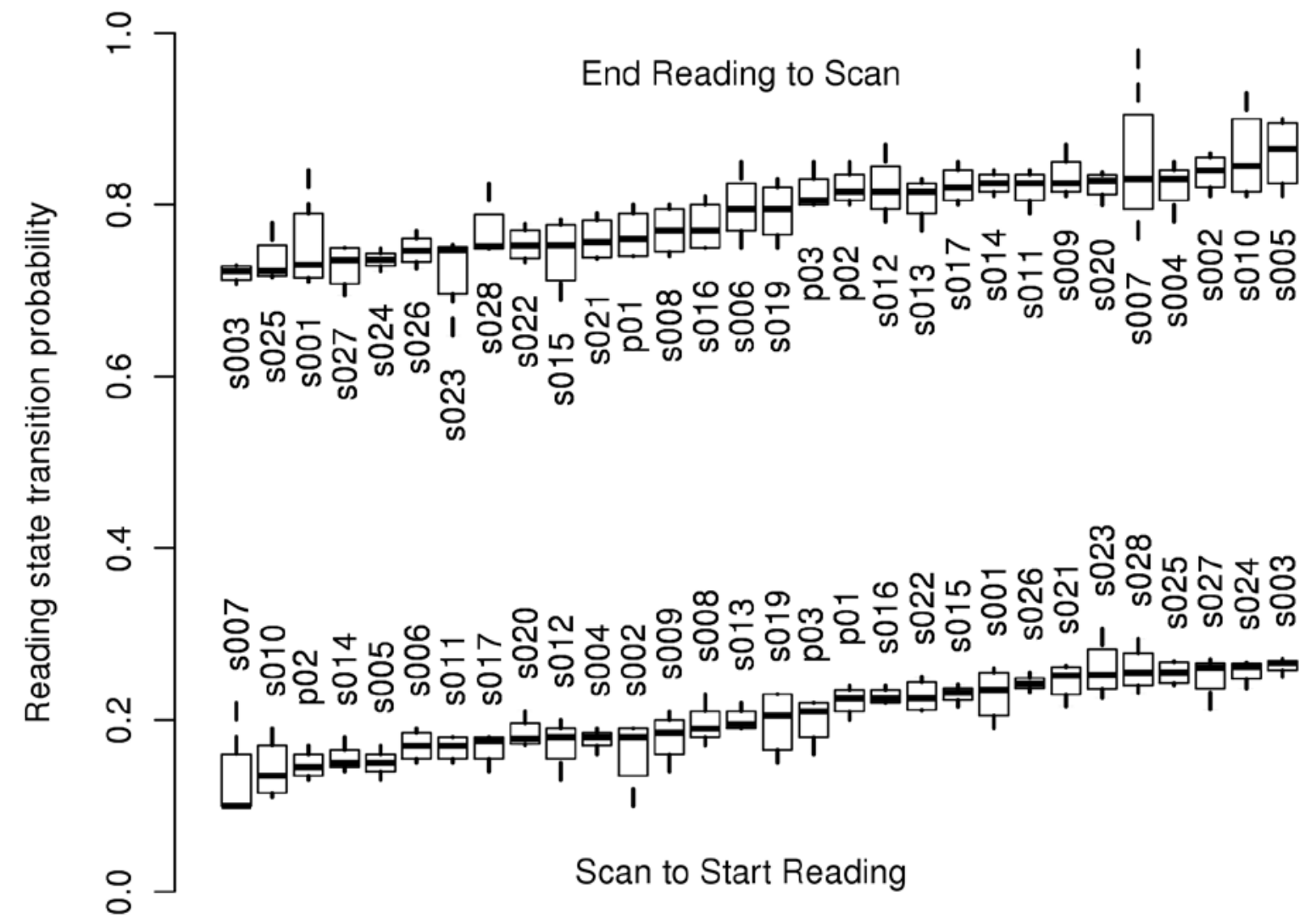

Figure 2: Individual differences in reading state transition probabilities.

The basic experiment model can be summarized as a causal chain where a user task induces a reading strategy, which produces the observed transition probabilities. We want to know if this causal chain is consistently similar across users.

Our tasks were developed to have one or more distinctive characteristics. A given task feature can be hypothesized to induce a change in the typical reading model by changing the transition probability for one or more nodes in the reading model. Changes from a user's typical reading model due to a task depends on some weighting of the influence of the task characteristics, and consequently the task demands on the user for successful task completion. The task characteristics in the classification system are categorical so for an initial analysis it is reasonable to suppose their influence is a weighted linear combination.

Ranking tasks by differences in the absolute changes in the state transition probabilities compared to each participant's typical reading model provides a description of the task influence on the model. Not having knowledge of the participant's typical model, we calculate an average reading model for each participant by summing the transition probabilities observed for the four tasks and taking the mean. The impact of each task was then calculated as the difference in the observed transition value from the average model mean. The participant's tasks were then ranked for each state transition in the model. For each state transition the set of task ranks was collected for all participants and Friedman's ANOVA rank sum test was applied to determine if there was a correlation between the tasks and the impact on individual reading models. Friedman's ANOVA requires balanced blocks. To compensate for the loss of two participants in the data, we performed the test on three blocks at a time (24 participants) over all combinations of balanced blocks. Table 2 reports the worst set of values obtained for selected reading model parameters. It shows that tasks affected the Scan to Start Reading and possibly the End Reading to Scan state transitions. The unreported parameters all had non-significant p-values.

Table 2:Friedman's ANOVA Test for Task Effects

\begin{tabular}{|c|c|c|c|}
\hline Reading Model Parameter & Friedman $\chi^{2}$ & df & p-value \\
\hline Scan to Start Reading & 15.1586 & 3 & 0.00169 \\
\hline Read to Read & 5.9359 & 3 & 0.11480 \\
\hline End Reading to Scan & 11.5385 & 3 & 0.00914 \\
\hline $\begin{array}{c}\text { Start Reading to End } \\
\text { Reading }\end{array}$ & 1.9258 & 3 & 0.58800 \\
\hline
\end{tabular}

Šidák correction $=0.013$ Bonferroni correction $=0.002$

Examination of the reading models for each participant reveals differences in the Scan to Start Reading probability for each task. It appears the AO and IP tasks induce many participants to switch from scanning to 

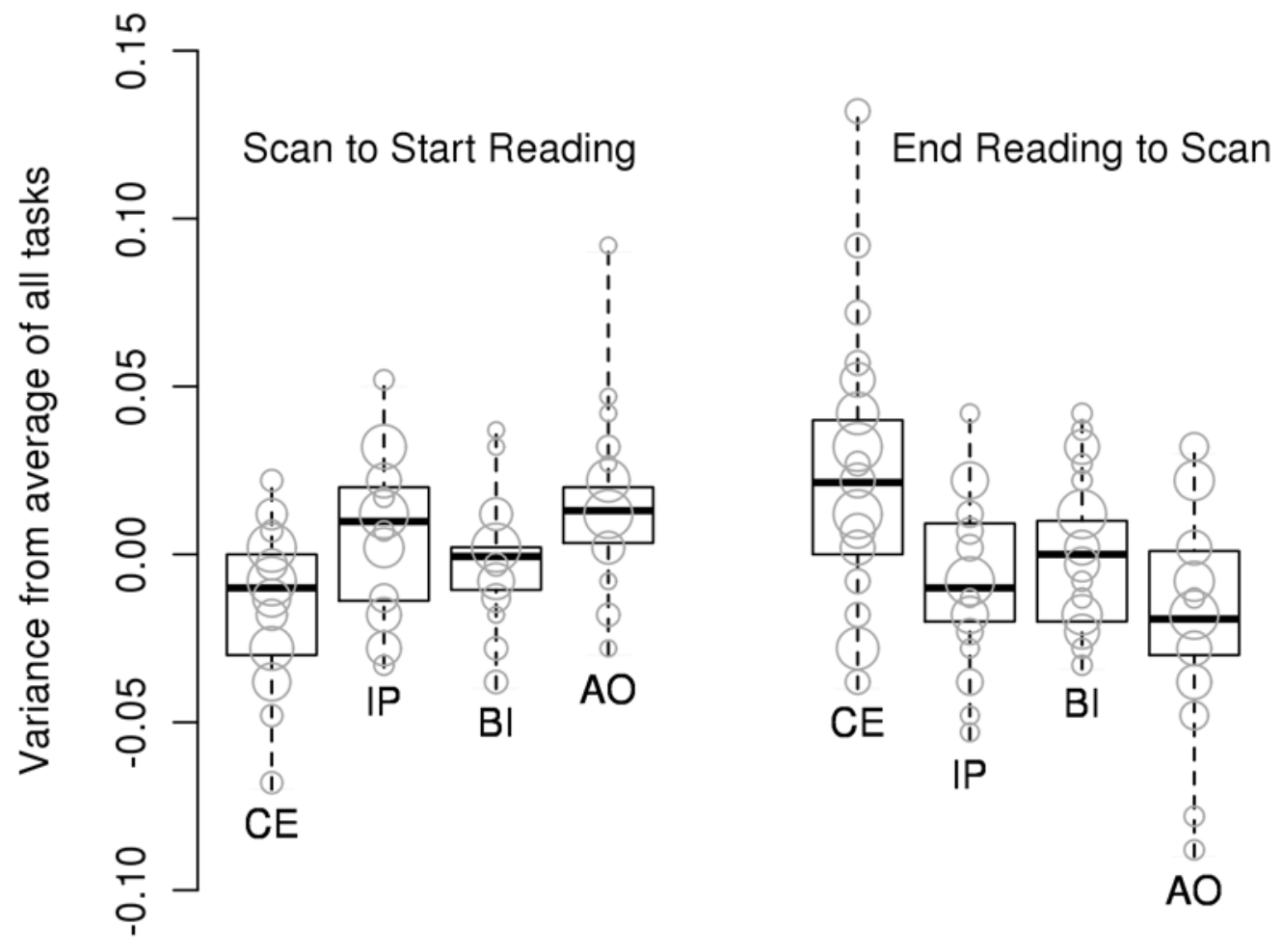

Figure 3: Task effects on reading state transition probabilities. The size of circles represents the distribution of the number of participants for each task.

reading more frequently. Likewise, the $\mathrm{CE}$ task is associated with a bias towards scanning. So AO and/or IP appear to bias many participants towards reading and CE appears to bias many participants towards scanning.

Figure 3 plots the variance in the Scan to Start Reading and End Reading to Scan transition probabilities from the average transition probability over all of the tasks for each user. Most participants were biased by AO (advanced obituary task) to more frequently switch from scanning to reading. For CE (fact checking task) most participants were biased to not switch to reading.

The End Reading to Scan transition occurs when the participant arrives at the end of a reading sequence and could transition to another reading sequence or switch to scanning (Figure 1). In those cases, the opposite behaviour was observed (Figure 3). For AO, the bias to switch to scanning was suppressed and they tended to continue to another reading sequence. For CE, participants were biased to switch to scanning. IP appears to have had an effect similar to AO, and BI had little impact.

\section{DISCUSSION}

The experiment was designed to examine task behaviour using realistic tasks in an unconstrained setting but in controlled conditions. Fixation patterns depend on expectations that in turn are conditioned on task properties. The calculated reading models revealed individual differences in their bias towards making the low-level decision to begin reading as an information acquisition strategy. Some participants had large variations by task and a few had strikingly little variation, but in most cases a similar range of values and a clear separation between each task's Scan to Start Reading transition probability was observed.

Recall that per the task classification, the AO and CE tasks were the most dissimilar. The task impact on reading models (Figure 3 ) shows distinct differences in the responses. For CE most participants made the decision to switch from scanning to reading less frequently and if they were reading they more frequently decided to switch back to scanning. Exactly the opposite behaviour is observed for AO.

AO and IP had a similar impact on the reading models. One could hypothesize the product and level task characteristics had more influence on the tendency to decide to switch from scanning to reading and to switch to a new reading sequence rather than scan after the end of a reading sequence. This hypothesis predicts the observed BI task impact on reading models, which is intermediate to the (AO, IP) tasks and the CE task.

\section{Applicability to Adaptive Systems}

These results may be straightforwardly applicable to user modelling for adaptive information systems. While the experiment setting was controlled, participants were free to go anywhere on the web to search for information to meet the task goal. The eye movement 
observations were of natural web search behaviour and subject to typical variations expected in operational environments -- web pages of various formats and types, with mixtures of text and images, some changing dynamically, for example advertisements, and so on. Even in such an unrestricted environment we were able to detect clear differences in the impact of the tasks on an individual's reading model. We expect even sharper distinctions in reading model differences when segments within each task session are examined.

The detection of reading model differences from eye movement patterns gathered over an entire session in a near operational environment suggests this approach to detecting tasks is robust. The reading model construction only requires analysis of the recent eye movement sequence to classify the observed fixations. So, although our analysis is based on entire sessions, the methodology allows for construction of reading models on the fly and real time detection of parameter changes.

This work supports the inference of a user's task from changes in reading models. It suggests real-time observations of eye movements can be used by adaptive search systems to incorporate task information into user models. This additional model dimension can be used to re-rank or filter search results, e.g. re-ordering search results more appropriately for the task, adapt information visualization, e.g. by providing more or less detail or different display modalities, and provide facilities to better support user's actions to complete their goal, e.g. suggest or trigger task-appropriate applications and tools.

\section{Limitations and Future Research}

One limitation in this analysis is the lack of baseline data about the reading models of individuals and the natural range in state transition probability variances. More work is needed to understand if there are useful baselines across classes of users, or if a user's typical reading model is an individual characteristic. Even if the absolute values of reading model parameters are individual characteristics, changes in reading model parameters as a function of task may still be associated with user types. This is another direction for future research.

In this work we looked at the search session-level impact of tasks. It seems likely users will exhibit changes in reading model parameters as they move from one interaction segment to another within a session. This work is being extended to look at changes in reading model parameters by segments within a session, where observed decisions by the participant, for example to reformulate a query or end the task, are used to define segments.

\section{CONCLUSIONS}

State transition models of the reading/scanning eye movements reveal important information about a user's cognitive processing of displayed information. At the task level, users apply search and processing strategies that depend upon properties of their primary search task.
In this work, we provide evidence for individual differences in reading models and for changes in an individual's visual cognitive processing strategy due to a high-level search task differences.

Reading is a key visual cognition strategy used to meet the needs of high-level information needs in complex cognitive tasks. While there is substantial work showing a link between immediate cognitive tasks and visual cognition strategies (Hayhoe et al. 2007, Triesch et al., 2007; Triesch et al., 2003), we are not aware of previous work showing effects of high-level information tasks on visual cognition strategies using reading models.

A general connection between information seeking tasks, at both high and low levels, and eye movement behaviour seems plausible whenever information retrieval interactions depend on a visual interface. User attention and cognitive resources, such as working memory, constrain information acquisition for each interaction as well as the information carried across interactions in the session. So a user's information seeking strategy, say a search strategy, affects the visual cognition strategy, e.g. an initial stance to scan rather than read a search results page, because of user expectations about the information environment they will process. Eye movements are a somewhat learnt behaviour (Hayhoe et al, 2007) and reflect this expectation.

While more research is needed to identify which task characteristics are responsible for reading model changes, our work suggests detection of reading models with eye tracking devices can reveal aspects of a user's current task. Unobtrusive task detection could enable information search and retrieval applications to adapt to changes in the cognitive information acquisition strategies of users as the search session unfolds.

\section{ACKNOWLEDGMENTS}

This work was supported by IMLS grant LM-06-070105-07.

\section{REFERENCES}

Belkin, N. (2008) Some(what) grand challenges for information retrieval. SIGIR Forum 42(1), 47-54.

Bierig, R., Cole., M.J., \& Gwizdka, J. (2009). A usercentered experiment and logging framework for interactive information retrieval. In N.J. Belkin, R. Bierig, G. Buscher, L. van Elst, J. Gwizdka, J. Jose, et al. (Eds), CEUR Workshop Proceedings: 512. Proceedings of the SIGIR 2009 Workshop on Understanding the User: Logging and interpreting user interactions in information search and retrieval, UIIR'2009 (pp. 8-11). Aachen, Germany: CEUR Workshop Proceedings.

Buscher, G., Dengel, A., \& Van Elst, L. (2008) Eye movements as implicit relevance feedback. In Proceedings of CHI '08 (Florence, Italy, 2008), ACM, pp. 2991-2998.

Buscher, G., Dengel, A., \& Van Elst, L. (2008) Query expansion using gaze-based feedback on the 
subdocument level. In Proceedings of SIGIR '08 (Singapore, July 2008), ACM, pp. 387-394.

Byström, K., \& Järvelin, K. (1995) Task complexity affects information seeking and use. Information Processing and Management 31(2), 191-213.

Conati, C., Merton, C., Muldner, K., \& Ternes, D. (2005) Exploring eye tracking to increase bandwidth in user modeling. In Proceedings of User Modeling 2005 (Edinburgh, Scotland, July 2005), L. Ardissomo, P. Brna, \& A. Mitrovic, Eds., Springer-Verlag, pp. 357-366.

Farzan, R., \& Brusilovsky, P. (2009) Social navigation support for information seeking. In UMAP 2009, Springer, pp. 66-77.

Findlay, J., \& Gilchrist, I. (2003) Active vision: The psychology of looking and seeing. Oxford University Press, New York, 2003.

Granka, L., Joachims, T., \& Gay, G. (2004) Eyetracking analysis of user behavior in WWW search. In Proceedings of SIGIR '04 (Sheffield, UK,,July 2004), ACM, New York, pp. 478-479.

Guan, Z., \& Cutrell, E. (2007) An eye tracking study of the effect of target rank on web search. In Proceedings of CHI '07 (San Jose, CA,USA, May 2007), ACM, p. 420.

Hayhoe, M.M., Droll, J., \& Mennie, N. (2007) Learning where to look. In R.P.G. van Gompel, M.H. Fischer, W.S. Murray, \& R.L. Hill, (Eds.) Eye movements: A window on mind and brain, ch. 30, pp. 642-659 Elsevier, New York..

Kelly, D., \& Belkin, N.J. (2004) Display time as implicit feedback: understanding task effects. In Proceedings of SIGIR '04 (Sheffield, UK, July 2004), ACM New York pp. 377-384.

Li, Y. (2009) Exploring the relationships between work task and search task in information search. Journal of the American Society for Information Science and Technology, 60(2), 275-291.

Liu, J. \& Belkin, N.J. (2010) Personalizing Information Retrieval for Multi-Session Tasks: The Roles of Task Stage and Task Type, In Proceedings of SIGIR '10 (Geneva, Switzerland, July 2010), ACM, New York.

Lorigo, L., Haridasan, M., Bryniarsodttir, H., Xia, L., Joachims, T., Gay, G., Granka, L., Pellacini, F., \& Pan, B. (2008) Eye tracking and online search: Lessons learned and challenges ahead. Journal of the American Society for Information Science and Technology, 59(7), 1041-1052.

Oliveira, F.T., Aula, A., \& Russell, D.M. (2009) Discriminating the relevance of web search results with measures of pupil size. In Proceedings of $\mathrm{CHI}$ '09 (Boston, MA, USA, April 2009), ACM, pp. 2209-2212.
Rayner, K., \& Fischer, M. (1996) Mindless reading revisited: Eye movements during reading and scanning are different. Perception \& Psychophysics 58(5), 734-747.

Rayner, K., Li, X., Williams, C.C., Cave, K.R., \& Well, A. (2007) Eye movements during information processing tasks: Individual differences and cultural effects. Vision Research 50(21), 27142726.

Rayner, K., Reichle, E.D., \& Pollatsek, A. (1998) Eye movement control in reading: An overview and model. In G. Underwood (Ed) Eye guidance in reading and scene perception, pp.243-268, Elsevier, Oxford.

Rayner, K., Smith, T.J., Malcomb, G.L., \& Henderson, J.M. (2009) Eye movements and visual encoding during scene perception. Psychological Science 20 (1), 6-10.

Rayner, K., White, S., Kambe, G., Miller, B., \& Liversedge, S. (2003) On the processing of meaning from parafoveal vision during eye fixations in reading. In R. Radach, J. Hyona, \& F. H. Deubel (Eds.) The mind's eye: Cognitive and applied aspects of eye movement research, pp. 213-234 Elsevier. New York.

Reichle, E.D., Pollatsek, A., \& Rayner, K. (2006) E-Z Reader: A cognitive-control, serial-attention model of eye-movement behavior during reading. Cognitive Systems Research 7(1), 4-22.

Reingold, E., \& Rayner, K. (2006) Examining the word identification stages hypothesized by the EZ Reader model. Psychological Science 17(9), 742746.

Sherman, C. (2005) A new f-word for Google search results. Search Engine Watch.

Terai, H., Saito, H., Egusa, Y., Takaku, M., Miwa, M., \& Kando, N. (2008) Differences between informational and transactional tasks in information seeking on the web. In Proceedings of IIIX '08 (London, UK, October 2008), ACM New York, pp. 152-159.

Torralba, A., Oliva, A., Castelhano, M., \& Henderson, J.M. (2006) Contextual guidance of eye movements and attention in real-world scenes: The role of global features in object search. Psychological Review 113 (4), 766-786.

Triesch, J., Ballard, D.H., Hayhoe, M.M., \& Sullivan, B.T. (2003) What you see is what you need. Journal of Vision 3(1), 86-94.

White, R.W., \& Kelly, D. (2006) A study on the effects of personalization and task information on implicit feedback performance. In Proceedings of CIKM '06 (Arlington, Virginia, USA, November 2006), ACM,

pp.

297-306. 